\title{
Postoperative long-term morbidity of extended endoscopic maxillectomy for inverted papilloma*
}

\author{
G. Bertazzoni', R. Accorona'1, A. Schreiber', G. Pietrobon², A. Karligkiotis², \\ E. Fazio ${ }^{2}$, P. Castelnuovo ${ }^{2}$, P. Nicolai ${ }^{1}$
}

Department of Otorhinolaryngology, University of Brescia, Brescia, Italy

Department of Otorhinolaryngology, University of Insubria, Varese, Italy
Rhinology 55: 319-325, 2017

https://doi.org/10.4193/Rhino17.035

*Received for publication:

February 21, 2017

Accepted: May 8, 2017

\begin{abstract}
Background: Extended endoscopic maxillectomy (Sturmann-Canfield procedure) allows full visualization of the maxillary sinus by sectioning the lacrimal duct and removing the medial part of the anterior maxillary wall. The aim of this study is to evaluate the morbidity of Sturmann-Canfield procedure in patients treated for inverted papilloma.
\end{abstract}

Methodology: The clinical records of all patients treated with a Sturmann-Canfield procedure for inverted papilloma from October 2000 to September 2015 at two teaching hospitals were reviewed. All patients were evaluated by nasal endoscopy and lacrimal system patency was assessed. Pre-maxillary cutaneous sensitivity was tested with a Semmes-Weinstein aesthesiometer and thermic stimulation. The SNOT-22 questionnaire was administered. Patients were also asked to report any other post-surgical complaints.

Results: Fifty-nine patients were identified. Mean follow-up after surgery was 66.3 months. Mean SNOT-22 score was 5.94 (range $0-20)$; the majority of patients (86\%) had a SNOT-22 symptom score $\leq 3$. Mucocoele occurred in $3(5 \%)$ cases. Lacrimal pathway obstruction was observed in 7 (12\%) patients. Fourteen (24\%) patients complained of paraesthesia in the malar area; hypoesthesia was present in only $5(8 \%)$ cases. Hypoesthesia in the region innervated by the anterior superior alveolar nerve was detected in 17 (29\%) patients. One patient reported a slight depression of paralateronasal soft tissues.

Conclusions: Although nasal function outcomes and the results from SNOT-22 questionnaires were favourable, a high rate of neurologic and lacrimal complications was observed. Potential morbidity of the intervention, including the possibility of negative aesthetic sequelae, should be discussed during preoperative counselling.

Key words: endoscopy, papilloma, inverted, maxillary sinus, morbidity, surgery, video-assisted

\section{Introduction}

Inverted papilloma (IP) of the maxillary sinus is currently treated primarily with endoscopic surgery ${ }^{(1)}$. Key elements for success are the correct identification and removal of the lesion pedicle along a sub-periosteal plane, with subsequent drilling of the underlying bone ${ }^{(2)}$. Endoscopic medial maxillectomy is performed to gain adequate exposure to the point of origin of the lesion, which is essential to avoid blind tearing of the pedicle (2). Accordingly, the extension of maxillary resection can be tailored to achieve proper exposure ${ }^{(2)}$. In order to appropriately address lesions originating from the lateral recess and/or the anterome- dial wall of the maxillary sinus, endoscopic maxillectomy can be extended by performing a Sturmann-Canfield procedure (SCP), which involves the removal of the entire medial wall of the maxillary sinus, sectioning of the nasolacrimal duct and enlargement of the pyriform aperture laterally up to the infraorbital foramen ${ }^{(2,3)}$. Although extended endoscopic maxillectomies, including SCP, have been validated in surgical practice ${ }^{(1,4-7)}$, data on long-term post-surgical morbidity and quality of life are lacking. The aim of the present study is to retrospectively evaluate the morbidity of SCP in a cohort of patients treated for IP of the maxillary sinus at two teaching hospitals. 


\section{Materials and methods}

A retrospective evaluation of clinical records of all patients treated with SCP for IP limited to the maxillary sinus at the Units of Otorhinolaryngology - Head and Neck Surgery of the Universities of Brescia and Insubria (Italy) from October 2000 to September 2015 was performed.

SCP was performed by removing the entire medial wall of the maxillary sinus together with the inferior turbinate, and sectioning the nasolacrimal duct at the junction with the lacrimal sac. The pyriform aperture was then enlarged laterally using a chisel and/or a diamond burr up to the area of the infraorbital foramen ${ }^{(2,3)}$. The bone underlying the insertion of the papilloma was drilled.

Patient evaluation included endoscopic examination of the sinonasal cavities and lacrimal pathway patency assessment with saline irrigation. The Sinonasal Outcome Test (SNOT-22) was administered to evaluate nasal function ${ }^{(8)}$.

Cutaneous hypoesthesia of the malar region was also assessed to identify the presence of impairment of the infraorbital nerve or one of its branches. Tactile sensitivity was tested with a 4.17 (1.2-1.6 g) Semmes-Weinstein monofilament aesthesiometer ${ }^{(9)}$. Cold sensitivity was tested by holding the finger of a latex glove full of iced water against the malar region. Heat sensitivity was evaluated using the tip of a steel spoon warmed in boiling water for 5 seconds and left for 15 seconds at room temperature until reaching $45^{\circ} \mathrm{C}$. Each malar area was tested twice for tactile, cold and heat sensitivity on the operated side, and then compared with the contralateral side. Patients were asked to report any sensitivity asymmetry or alterations between the two sides. All patients were also asked to report any other post-surgical complaint; the presence and relevance of possible aesthetic sequelae were investigated.

The study was conducted according to the principles of the revised Declaration of Helsinki, in compliance with Good Clinical Practice and ethical standards and was approved by respective local Ethics committees (Comitato Etico Provinciale della Provincia di Brescia and Comitato Etico Provinciale di Varese).

\section{Results}

Fifty-nine patients ( 43 males and 16 females) were identified. Mean age at the time of surgery was $57.9 \pm 15$ years (median 60 ), and mean follow-up after surgery was $66.3 \pm 47$ months (median 53 months), ranging from 10 months to 190 months. Thirty-two patients underwent primary surgery, while 27 were revision cases. At last follow-up, 56 patients were recurrencefree, 3 patients underwent further surgery for recurrence located in the maxillary sinus. Revision surgery after SCP was of
Table 1. Results of SNOT-22 questionnaire in 48 patients undergoing SCP.

\begin{tabular}{|lcc|}
\hline \multicolumn{1}{|c}{ Symptom } & $\begin{array}{c}\text { Median score } \\
\text { (range) }\end{array}$ & $\begin{array}{c}\text { Number of } \\
\text { patients (\%) }\end{array}$ \\
\hline Need to blow the nose & $0(0-4)$ & $19(40 \%)$ \\
\hline Sneezing & $0(0-2)$ & $12(25 \%)$ \\
\hline Rhinorrhea & $0(0-4)$ & $14(29 \%)$ \\
\hline Cough & $0(0-4)$ & $6(12 \%)$ \\
\hline Post-nasal drip & $0(0-5)$ & $12(25 \%)$ \\
\hline Thick nasal discharge & $0(0-3)$ & $17(35 \%)$ \\
\hline Ear fullness & $0(0-2)$ & $6(12 \%)$ \\
\hline Dizziness & $0(0-1)$ & $3(6 \%)$ \\
\hline Ear pain/pressure & $0(0-1)$ & $3(6 \%)$ \\
\hline Facial pain/pressure & $0(0-3)$ & $7(14 \%)$ \\
\hline Difficulty falling asleep & $0(0-3)$ & $4(8 \%)$ \\
\hline Waking up at night & $0(0-3)$ & $8(16 \%)$ \\
\hline Lack of good night sleep & $0(0-3)$ & $10(21 \%)$ \\
\hline Waking up tired & $0(0-3)$ & $7(14 \%)$ \\
\hline Fatigue during the day & $0(0-1)$ & $3(6 \%)$ \\
\hline Reduced productivity & $0(0-1)$ & $2(4 \%)$ \\
\hline Reduced concentration & $0(0-1)$ & $2(4 \%)$ \\
\hline irritability & $0(0-2)$ & $4(8 \%)$ \\
\hline Sadness & $0(0-1)$ & $3(6 \%)$ \\
\hline Embarrassment & $0(0)$ & $0(0 \%)$ \\
\hline Loss of taste/smell & $0(0-4)$ & $13(27 \%)$ \\
\hline Nose congestion & $0(0-2)$ & $14(29 \%)$ \\
\hline
\end{tabular}

minimal extent in all 3 cases, since all recurrences were timely detected at an early stage during postoperative endoscopic follow-up. All patients underwent submucosal removal of the recurrent IP with drilling of the underlying bone. There was no need to further extend the anterior maxillary resection performed at primary surgery.

\section{Quality of life and nasal function}

Forty-eight patients completed the SNOT-22 questionnaire; the results are summarised in Table 1. Mean and median SNOT22 score were 5.94 (range 0-20) and 4, respectively. The most frequently reported symptoms were need to blow the nose (40\%) and thick nasal discharge (35\%) (Table 1); the majority of patients (86\%) had minor or no nasal complaints (SNOT-22 symptom score $\leq 3$ ), the median score for all symptoms was 0 . During the early postoperative period, all patients were treated with high volume/low pressure saline nasal irrigations and 


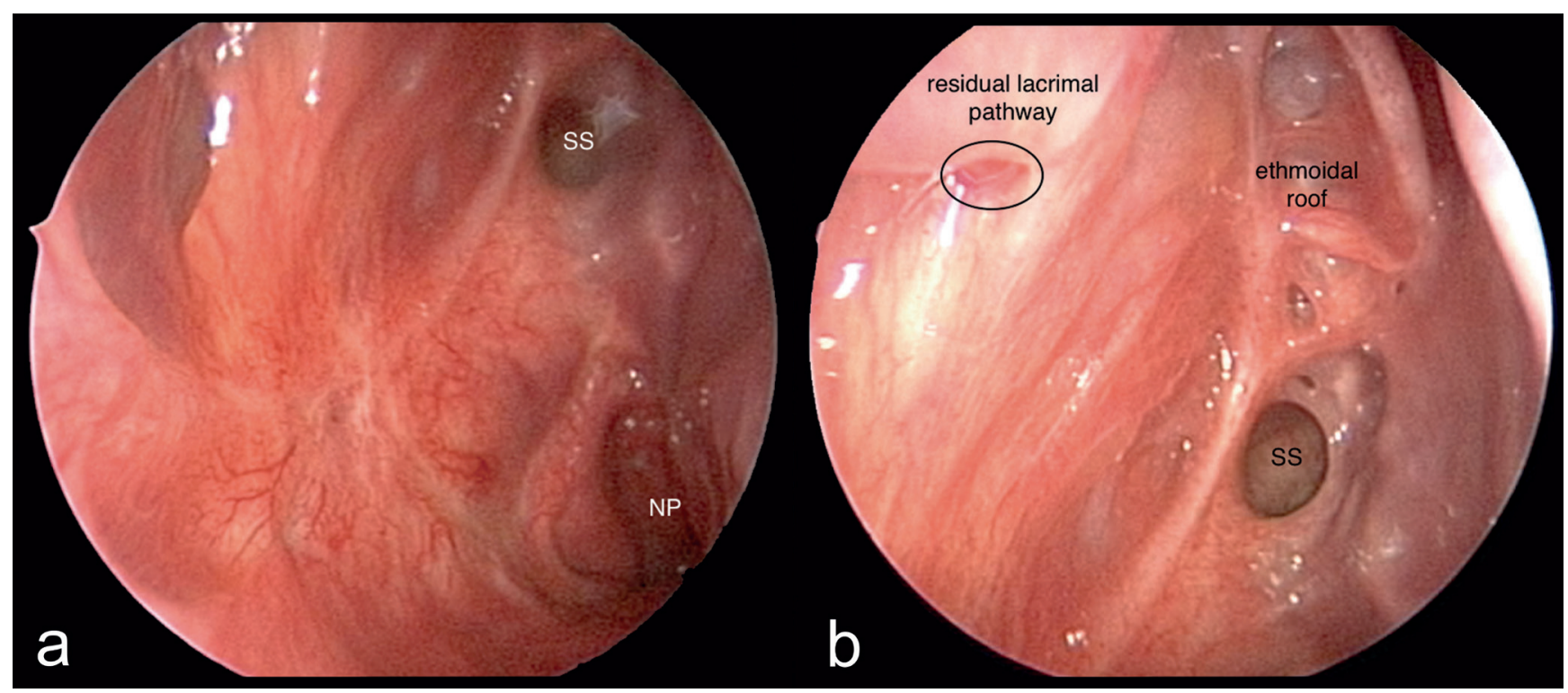

Figure 1. Post-operative endoscopic pictures at 14 months: scarring and shrinkage of the surgical cavity are evident (a). Nasopharynx (NP); sphenoid sinus (SS). Lacrimal pathway (black circle) and ethmoid roof appearance (b) are also visible.

Vaseline-based nasal ointments at least three times a day. At last follow-up, all patients had reduced the frequency of nasal irrigation to once a day and no longer used ointments. In all cases, endoscopic evaluation of the nose revealed well remucosised surgical cavities and absence of crusting (Figure 1). A common feature in all patients was cicatricial shrinkage of the surgical cavity with substantial reduction of the volume of the maxillary sinus (Figure 1 and 2).

In 3 (5\%) cases a mucocoele occurred, and all patients were effectively treated with endoscopic surgical marsupialisation (2 cases under general anaesthesia and 1 under local anaesthesia).

\section{Lacrimal pathway obstruction}

Post-operative lacrimal pathway obstruction occurred in 7 (12\%) patients, and was successfully managed with endoscopic dacryocystorhinostomy (endo-DCR) under general anaesthesia in 6 cases. One patient, who had mild and intermittent epiphora, refused revision surgery.

\section{Sensory nerve damage}

Cutaneous hypoesthesia in the malar area, related to infraorbital nerve damage, was present in 5 (8\%) patients. Tactile hypoesthesia was demonstrated in all 5 cases using the SemmesWeinstein aesthesiometer; only 2 (3\%) also reported thermic hypoesthesia when tested for cold and heat sensitivity. When asked to report any other long-term post-surgical complaints, 14 (24\%) patients complained of paraesthesia of variable entity in the malar area, while 17 (29\%) patients referred persistent hypoesthesia of the anterior superior dental hemi-arch on the operated side, in the area mainly supplied by the anterior superior alveolar nerve (ASAN).

The distress caused by malar hypoesthesia, paraesthesia and dental hypoesthesia was mild in all cases, and none of the patients had previously mentioned the problem during follow-up visits, as it was not deemed relevant.

\section{Aesthetic outcomes}

One patient reported a slight depression of paralateronasal soft tissues on the operated side. The defect was barely noticeable on close inspection and the patient was not bothered by it, nor did he consider the defect aesthetically relevant.

\section{Discussion}

Our retrospective study is the first to carry out combined assessment of sinonasal and lacrimal function, local sensitivity and aesthetic alterations to provide a comprehensive evaluation of postoperative long-term morbidity and quality of life after SCP. The study reviews 59 patients and, to the best of our knowledge, is the largest retrospective analysis on long-term morbidity of extended endoscopic maxillectomies to date. A strength of the study is the homogeneity of the cohort, which includes only patients treated for a specific disease whose dissection was confined to the maxillary sinus.

While our results on nasal morbidity are in agreement with previous experiences ${ }^{(10,11)}$, we found a significant rate of neurological and lacrimal complications. On the other hand, although it is not the main focus of the study, our series confirms the favourable control of disease achieved by SCP, with $95 \%$ of patients free of disease after a mean follow-up of 62.5 months. Our long-term results compare favourably with the experience of Kamel, who 


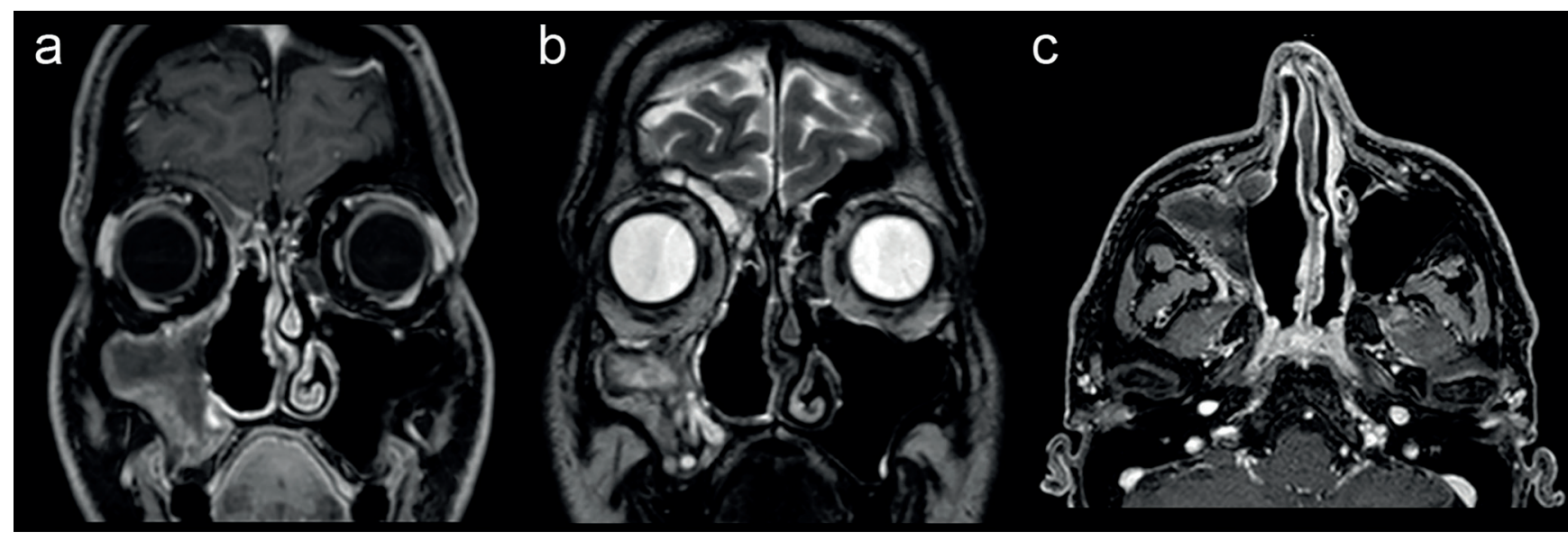

Figure 2. Post-operative MRI at 14 months ( $\mathrm{T} 1$ coronal (a), $\mathrm{T} 2$ coronal (b), $\mathrm{T} 1$ axial (c)) shows scar tissue in the surgical cavity and the size of the air passage in the operated nasal fossa.

reported a $15 \%$ recurrence rate in a group of 51 patients with maxillary sinus IP treated with endoscopic medial maxillectomy after 8.8 years of follow-up ${ }^{(12)}$. Results more similar to ours were achieved by Adriaensen et al., who described 5 recurrences ( 3 in the same patient) in a series of 61 patients with maxillary sinus IP with a follow up of 35.5 months for primary IP and 45.1 months for revision IP (13).

\section{Quality of life and nasal function}

Quality of life was minimally affected by SCP, as demonstrated by SNOT-22 results. Our median SNOT-22 score ${ }^{(4)}$, which is 5.3 points lower than the average score of healthy people (9.3) ${ }^{(14)}$, reflects an optimal quality of life. However, we do not believe that our results correspond to a genuine improvement in quality of life compared to the general population, as it is known that changes of less than 9 points in SNOT-22 score cannot be perceived as worsening/improvement ${ }^{(8)}$. Our lower median score could be explained by the small size of our study population or selection bias related to age and gender, since most patients (59\%) were males over the age of 50 years.

Previous studies on quality of life after resection of sinonasal tumours performed with SNOT-22 or SNOT-20 showed results that were not significantly different from the healthy population (10,11). Harrow and Batra performed a retrospective long-term study on minimally-invasive resection of sinonasal tumours, which included 32 patients treated for IP, and showed that the mean postoperative SNOT-20 score was lower than the mean score in healthy individuals after 24 months ${ }^{(11)}$. However, a comparison with our results is difficult, because IP location and surgical treatment were not described by the authors and the mean follow-up was shorter ( 6 months). The only study that specifically focused on long-term quality of life after resection of IP reported a median SNOT-22 score of $12{ }^{(10)}$, which does not significantly deviate from the score in healthy individuals ${ }^{(8)}$. These results are not entirely comparable to ours, as that study
(10) analysed patients with IP originating from different sites and included only 5 patients treated with endoscopic medial maxillectomy. Nonetheless, this report indicates very modest, if at all, alterations in quality of life after surgery for IP. The most frequent symptom in this study was the need to blow the nose (median score 1) ${ }^{(10)}$. Other frequent symptoms were waking at night, postnasal discharge, thick nasal discharge and sneezing ${ }^{(10)}$. Thick nasal discharge, which was frequently reported both in our series and the study by van Samkar and Georgalas ${ }^{(10)}$, is allegedly related to the disruption of the mucociliary epithelium caused by post-surgical scarring. The other frequently reported symptoms can be attributed to the change of airflow caused by a larger nasal cavity.

Considering the extent of the resection in SCP, which includes the inferior turbinate, greater long-term impairment of nasal function could be expected with higher SNOT-22 scores. However, cicatricial shrinkage of the maxillary sinus with significant reduction in the volume of the surgical cavity was observed in all cases (Figure 1 and 2), a finding which could favourably impact crusts formation and prevent empty nose syndrome.

\section{Lacrimal pathway obstruction}

Complications related to lacrimal drainage after endoscopic medial maxillectomy have been previously addressed in the literature and a variable rate of obstruction (0-16\%) has been reported ${ }^{(15-18)}$. All studies included a small number of patients (ranging from 6 to 17) who had the lacrimal pathway managed in different ways ${ }^{(15-18)}$. In a prospective study performed by Sadeghi and Joshi, the authors compared two groups of patients undergoing endoscopic maxillectomy with sectioning of the nasolacrimal duct: five patients underwent simple nasolacrimal duct dissection and 7 underwent DCR with Jones silastic stent placement ${ }^{(15)}$. No patient developed epiphora after a minimum one-year follow-up ${ }^{(15)}$. Similarly, a retrospective study by Imre 
et al. on 12 patients who underwent only nasolacrimal duct section reported no lacrimal pathway obstruction after a mean follow-up of 21.1 months ${ }^{(16)}$. Both studies, albeit on a small number of patients, concluded that concurrent DCR does not seem necessary to preserve the patency of the lacrimal pathway ${ }^{(16)}$. Differently, a retrospective study on 17 patients who underwent nasolacrimal duct section reported a $6 \%$ rate of postoperative epiphora ${ }^{(17)}$. Furthermore, in another retrospective study on 6 patients treated with endoscopic medial maxillectomy and DCR with placement of a bicanalicular silicone stent, 1 (16\%) patient postoperatively complained of intermittent epiphora ${ }^{(18)}$.

Overall, data from the available literature do not clearly indicate optimal management of the lacrimal pathway during extended endoscopic maxillectomy, and further prospective studies are needed.

Given the rate of lacrimal pathway obstruction in our series, performing endoscopic DCR during SCP might decrease the occurrence to a rate around $7 \%{ }^{(19,20)}$. Indeed, the impact of endoscopic DCR on SCP morbidity deserves further prospective investigation.

On the other hand, variations of extended endoscopic maxillectomy with lacrimal duct preservation are undeniably appealing, but should be employed without compromising adequate visualisation of the insertion of the lesion and taking into account variations in anatomy between the anterior maxillary wall and the lacrimal duct system ${ }^{(21,22)}$.

\section{Sensory nerve damage}

Malar sensory alterations are related to damage of branches of the infraorbital nerve during dissection of the premaxilla soft tissues that is performed to expose the bone lateral to the pyriform aperture, reaching the infraorbital foramen as the limit of the resection. Indeed, it is possible that damage of small nerve fibres may only produce paraesthesia, as reported in the majority of cases, while hypoesthesia can be related to damage of major branches. Larger divisions of the infraorbital nerve may be harder to identify when they branch out from an accessory foramen medial to the infraorbital foramen, which can be present in $0.8 \%$ to $27.3 \%$ of cases ${ }^{(23)}$. The majority of accessory infraorbital foramina are located in the superomedial side of the infraorbital foramen (92.2\%), while a small proportion (7.8\%) is located inferomedially, in the area of bone that is drilled during SCP (23). Damage could occur from direct lesion of the accessory branch or by thermal damage caused by drilling the adjacent bone. The low prevalence of accessory inferomedial branches of the infraorbital nerve could explain the variability of nerve damage caused by SCP and the small proportion of patients with hypoesthesia in our series. Preoperative CT identification of accessory foramina or increased awareness of their presence could reduce sensory damage in the malar region related to SCP. Hypoesthesia of the anterior superior dental hemi-arch on the operated side is related to ASAN damage. It is plausible that the ASAN was injured during enlargement of pyriform aperture, even if most patients (76\%) did not experience any sensory impairment. Essentially, dental sensitivity was preserved thanks to the complex anastomotic network that connects the anterior, middle, and posterior alveolar nerves ${ }^{(24)}$.

Although ASAN hypoesthesia was the most common long term sequela of SCP, no patient reported that quality of life was af-

fected. Nonetheless, this frequent sequela should be addressed preoperatively during informed consent discussion.

Overall, our evaluation of postoperative sensory alterations, by analysing all the main nerves involved in the surgical field, provides a comprehensive picture of all possible sensory sequelae of SCP. However, our findings are not entirely based on objective measures, and mostly rely on descriptive findings. Indeed, only tactile and thermal sensitivity in the malar area were formally tested, while malar paraesthesia and ASAN hypoesthesia were evaluated based on symptoms reported by patients. Nonetheless, our evaluation was able to clarify that sensory alterations after SCP had a minimal impact on quality of life.

\section{Aesthetic outcomes}

All patients were asked to report unfavourable aesthetic outcomes related to pyriform aperture resection, which theoretically could reduce the support of overlying soft tissues and cause ala nasi depression ${ }^{(25)}$.

Although this complication was rare in our series (1.7\%) and was not related to significant aesthetic sequelae, the possibility of more marked defects with unfavourable outcomes should be considered before SCP and discussed with the patient. The occurrence of this particular sequela has not been previously addressed in the literature and speculation can only be made on the mechanisms underlying this event ${ }^{(25)}$. Indeed, it is possible that a combination of reduced soft tissue support and scarring retraction are involved in collapse of the area lateral to the nose. When feasible, less extensive drilling of the pyriform aperture could reduce the possibilities of unfavourable aesthetic outcomes. Wide exposure of the antero-lateral wall of the maxillary sinus can be also obtained with transseptal approaches ${ }^{(26,27)}$. Further prospective studies with objective evaluation of facial asymmetry are needed to better understand the pros and cons of different approaches.

\section{Additional considerations}

A review of the literature on endoscopic maxillectomies highlights the lack of a clear, univocal and consistent nomenclature to describe the wide array of procedures collectively described as extended endoscopic maxillectomies. In general, this term identifies any procedure on the medial wall of the maxillary sinus that is extended beyond a wide middle antrostomy, involving other structures such as the inferior turbinate, the lacrimal 
pathway, or the pyriform aperture. The first description of the endoscopic transnasal removal of all the mentioned structures was made by Brors and Draf ${ }^{(3)}$, who performed the intervention under endoscopic guidance, following what had already been performed under direct view by Sturmann and Canfield at the beginning of the 20th century ${ }^{(28,29)}$. Conversely, removal of the entire wall of the maxillary sinus through a sublabial approach, leaving the pyriform aperture intact, was described by Denker ${ }^{(30,31)}$. Even though the procedure was sublabial, the term endoscopic Denker procedure has become popular to indicate the removal of the entire maxillary wall with a transnasal endoscopic approach ${ }^{(1,25,27)}$. However, only the name SCP clearly identifies a transnasal procedure that removes the entire medial maxillary wall with inferior turbinate and enlarges the piriform aperture, with sectioning of the lacrimal pathway ${ }^{(3)}$. A recent study on extended endoscopic maxillectomies proposed a uniform nomenclature for the various technical variations of this procedure, including SCP (32). Indeed, adhering to one classification would make it easier to compare the results of different groups.

\section{Conclusion}

Although nasal function outcomes and the results from SNOT-22 questionnaires were favourable, a high rate of neurologic and lacrimal complications was observed after SCP. However, sensory alterations did not significantly affect patient quality of life and lacrimal pathway obstruction can be resolved with DCR. Nonetheless, potential morbidity of the intervention, including the possibility of negative aesthetic sequelae, should be discussed with the patient during preoperative counselling.

\section{Authorship contribution}

GB contributed to the design of the study, wrote the work, and contributed to data collection and analysis, RA contributed to the design of the study, data collection and analysis, and manuscript draft revision, GP contributed to data collection and analysis, AK contributed to data collection and analysis, EF contributed to data collection and analysis, AS contributed to the design of the study and supervised the work, PC supervised the work, provided pictures, and reviewed manuscript drafts, PN contributed to the design of the study, supervised the work, guided the bibliographic research, and reviewed manuscript drafts.

\section{Conflict of interest}

No conflict of interest exists.

\section{References}

1. Carta F, Blancal JP, Verillaud B, et al. Surgica management of inverted papilloma: approaching a new standard for surgery. Head Neck 2013; 35: 1415-1420.

2. Tomenzoli D, Castelnuovo P, Pagella F, et al. Different endoscopic surgical strategies in the management of inverted papilloma of the sinonasal tract: experience with 47 patients. Laryngoscope 2004; 114: 193-200.

3. Brors D, Draf W. The treatment of inverted papilloma. Curr Opin Otolaryngol Head Neck Surg 1999; 7: 33.

4. Busquets JM, Hwang PH. Endoscopic resection of sinonasal inverted papilloma: a meta-analysis. Otolaryngol Head Neck Surg 2006; 134: 476-482

5. Minovi A, Kollert M, Draf W, Bockmuhl U. Inverted papilloma: feasibility of endonasa surgery and long-term results of 87 cases. Rhinology 2006; 44: 205-210.

6. Lombardi D, Tomenzoli D, Buttà $L$, et al. Limitations and complications of endoscopic surgery for treatment for sinonasal inverted papilloma: a reassessment after 212 cases. Head Neck 2011; 33: 1154-1161.

7. Pagella F, Giourgos G, Matti E, Canevari FR, Carena P. Endoscopic treatment of maxillary inverted papilloma. Rhinology 2011; 49: 369-374.

8. Hopkins C, Gillett S, Slack R, Lund VJ, Browne JP. Psychometric validity of the 22-item Sinonasal Outcome Test. Clin Otolaryngol 2009; 34: 447-454.

9. Bell-Krotoski J, Weinstein S, Weinstein C Testing sensibility, including touch-pres sure, two-point discrimination, point locali- zation, and vibration. J Hand Ther 1993; 6: 114-123.

10. van Samkar A, Georgalas C. Long-term quality of life after endoscopic removal of sinonasal inverted papillomas: a 6-year cohort analysis in a tertiary academic hospital. Eur Arch Otorhinolaryngol 2016; 273: 1433 1437

11. Harrow BR, Batra PS. Sinonasal quality of life outcomes after minimally invasive resection of sinonasal and skull-base tumors. Int Forum Allergy Rhinol 2013; 3: 1013-1020.

12. Kamel RH, Abdel Fattah AF, Awad AG. Transnasal endoscopic medial maxillectomy in recurrent maxillary sinus inverted papilloma. Rhinology. 2014; 52: 381-5.

13. Adriaensen GF, Lim KH, Georgalas C, Reinartz SM, Fokkens WJ. Challenges in the Management of Inverted Papilloma: A Review of 72 Revision Cases. Laryngoscope 2016; 126: 322-8.

14. Gillett S, Hopkins C, Slack R, Browne JP. A pilot study of the SNOT 22 score in adults with no sinonasal disease. Clin Otolaryngol 2009; 34: 467-469.

15. Sadeghi N, Joshi A. Management of the nasolacrimal system during transnasal endoscopic medial maxillectomy. Am J Rhinol Allergy 2012; 26: 85-88.

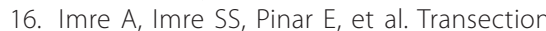
of Nasolacrimal Duct in Endoscopic Medial Maxillectomy: Implication on Epiphora. J Craniofac Surg 2015; 26: 616-619.

17. Liu Q, Yu H, Minovi Aet al. Management of maxillary sinus inverted papilloma via transnasal endoscopic anterior and medial maxillectomy. ORL J Otorhinolaryngol Relat
Spec 2010; 72: 247-251.

18. Eloy P, Mardyla N, Bertrand B, Rombaux P. Endoscopic endonasal medial maxillectomy: case series. Indian J Otolaryngol Head Neck Surg 2010; 62: 252-257.

19. Jung SK, Kim YC, Cho WK, Paik JS, Yang SW. Surgical outcomes of endoscopic dacryocystorhinostomy: analysis of 1083 consecutive cases. Can J Ophthalmol 2015; 50: 466470.

20. Ali MJ, Psaltis AJ, Murphy J, Wormald PJ. Powered endoscopic dacryocystorhinostomy: a decade of experience. Ophthal Plast Reconstr Surg 2015; 31: 219-221.

21. Nakamaru Y, Furuta Y, Takagi D, Oridate $N$, Fukuda S. Preservation of the nasolacrimal duct during endoscopic medial maxillectomy for sinonasal inverted papilloma. Rhinology 2010; 48: 452-456.

22. Simmen $D$, Veerasigamani $N$, Briner $H R$ Jones N, Schuknecht B. Anterior maxillary wall and lacrimal duct relationship - CT analysis for prelacrimal access to the maxillary sinus. Rhinology. 2017 Jun 1;55(2):170174

23. Hwang K, Lee SJ, Kim SY, Hwang SW Frequency of existence, numbers, and location of the accessory infraorbital foramen. J Craniofac Surg 2015; 26: 274-276.

24. Robinson S, Wormald PJ. Patterns of innervation of the anterior maxilla: a cadaver study with relevance to canine fossa puncture of the maxillary sinus. Laryngoscope 2005; 115: 1785-1788

25. Prosser JD, Figueroa R, Carrau RI, Ong YK, Solares CA. Quantitative analysis of endoscopic endonasal approaches to the 
infratemporal fossa. Laryngoscope 2011; 121: 1601-1605.

26. Dean NR, Illing EA, Woodworth BA. Endoscopic resection of anterolateral maxillary sinus inverted papillomas. Laryngoscope 2015; 125: 807-812.

27. Upadhyay S DR, Buohliqah L, Fiore ME et al. Effect of Incremental Endoscopic Maxillectomy on Surgical Exposure of the Pterygopalatine and Infratemporal Fossae. J Neurol Surg B Skull Base 2016; 77: 66-74.

28. Sturmann D. Die intranasale Eröffnung der Kieferhöhle. Berl Klin Wochenschr 1908; 27 1273-1274.

29. Canfield R. The submucous resection of the lateral nasal wall in chronic empyema of the antrum, ethmoid and sphenoid. JAMA 1908; 51: 1136-1141.

30. Denker A. Ein neuer weg fur die operation der malignen nasentumoren. Munch Med Wochenschr 1906; 53: 953-956.

31. Sanderson RJ, Knegt P. Management of inverted papilloma via Denker's approach. Clin Otolaryngol Allied Sci 1999; 24: 69-71.

32. Schreiber A, Ferrari M, Rampinelli V et al. Modular Endoscopic Medial Maxillectomies: Quantitative Analysis of Surgical Exposure in a Preclinical Setting. World Neurosurg. 2017 Apr;100:44-55.
Giacomo Bertazzoni, MD Dept. of Otorhinolaryngology University of Brescia Piazzale Spedali Civili 1

25123 Brescia

Italy

Tel: +39-30-399 5322

Fax: +39-30-399 5038

E-mail: ilbertaz@gmail.com 